\title{
Comportamiento informacional y políticas públicas de información: consideraciones teóricas alrededor del caso de DATASUS en Brasil
}

\author{
Cristian Berrío Zapata * \\ Fernando de Assis Rodrigues \\ Rita de Cássia Cassiano Lopes \\ Angela Maria Grossi de Carvalho \\ Ricardo Cesar Gonçalves Santana **
}

Artículo recibido:

26 de septiembre de 2014.

Artículo aceptado:

9 de febrero de 2016.

\section{Resumen}

La era de la información necesita políticas de información para la articulación global; Brasil propuso políticas que, a pesar de sus buenas intenciones, no consiguen los resultados deseados. Es el caso de DATASUS, sistema de información de salud brasileño. Tales políticas ignoran los descubrimientos de la ciencia de información sobre la naturaleza del sujeto informacional. Este artículo discute algunos modelos y teorías sobre el comportamiento informacional que deberían ser considerados en los debates sobre políticas de informa-

* Universidade Federal do Pará, Instituto de Ciências Sociais Aplicadas ICSA, Pará-Brasil. berriozapata@ufpa.br

** Los autores pertenecen a la UNESP-Universidade Estadual Paulista, São PauIo-Brasil. (fernando@elleth.org); (ritacassiano@marilia.unesp.br); (angela@faac. unesp.br); (ricardosantana@tupa.unesp.br)

INVESTIGACIÓN BIBLIOTECOLÓGICA, Vol.30, Núm.69, mayo/agosto, 2016, México, ISSN: 0187-358X. pp. 19-41 
ción, y plantea un modelo de "Campo Informacional" con temáticas que pueden guiar su construcción.

Palabras clave: Políticas de Información; Internet; Sistemas de Información; e-Government; Brasil.

\section{Abstract}

Information behavior and public policies for information: theoretical considerations regarding DATASUS in Brazil

Cristian Berrio-Zapata, Fernando de Assis-Rodrigues, Rita de Cássia Cassiano-Lopes, Angela Maria Grossi de Carvalho and Ricardo Cesar Gonçalves-Santana

The information age requires information policies to guide its global articulation. Brazil has attempted to answer this call with policies that despite their good intentions have not obtained desired results. DATASUS, the Brazilian health information system, operates under policies that seem to be designed without considering the latest discoveries of Information Science regarding the informational nature of humans. This article discusses several information behavior models and theories that must be considered when formulating information policies, and proposes an "Information Field" model containing guideposts for their construction.

Keywords: Information Policy; Internet; Information Systems; e-government; Brazil.

\section{INTRODUCCIÓN}

En la era de la información el discurso sobre la necesidad ciudadana de la punta del iceberg. El reto incluye evaluar empíricamente los flujos informacionales en la población, sus efectos, e identificar los problemas que afectan su democratización. A partir de la década de 1990 se diseñaron en Brasil y otros países de Latinoamérica múltiples políticas de información, y aun cuando la tendencia reduccionista de estas propuestas ha venido cediendo, 
su diseño todavía se hace de espaldas a los avances de la ciencia de la información en tres aspectos:

1. Se persiste en mantener una perspectiva centrada en "el sistema", abandonando al usuario.

2. Se ignoran los últimos modelos y teorías sobre el comportamiento informacional humano.

3. Se mantiene una perspectiva racional-reduccionista sobre una visión ecológica y de contexto.

Este artículo discute varios modelos y teorías sobre comportamiento informacional y su relación con el desarrollo de políticas de información, partiendo de los problemas reportados en los sistemas de información pública, ejemplificados con el caso del sistema de información de salud brasileño DATASUS. Se describen las normas que dieron origen a DATASUS y las debilidades que fueron identificadas en él por medio de estudios empíricos. Como conclusión, se propone un mapa conceptual del "campo informacional" que afecta a las políticas de información alrededor del ejemplo de DATASUS, para alimentar su debate y mejorar la fundamentación de políticas de este tipo.

\section{TRANSPARENCIA, DATOS ABIERTOS Y DATASUS}

Las políticas de información se apoyan en el acceso abierto y la transparencia de los datos. ${ }^{1}$ La transparencia marca un estilo de gobernanza que redistribuye la coordinación de recursos y competencias entre los órdenes institucionales y organizacionales, públicos y privados, abandonando el modelo de monopolio estatal y buscando pluralismo en las funciones públicas. ${ }^{2}$ Las tecnologías informáticas pueden ampliar el acceso a datos, intensificar la demanda de informaciones y estimular la transparencia en el Estado, produciendo nuevas formas de interacción Estado-ciudadanía. ${ }^{3}$ El acceso a datos gubernamentales es condición para la participación ciudadana en los procesos políticos y de gestión pública que efectivizan la democracia. ${ }^{4}$ Internet

1 R. C. G. Sant'ana, Tecnologia e gestão pública municipal : mensuração da interação com a sociedade.

2 A. M. B. Malin, "Gestão da informação governamental: em direção a uma metodologia de avaliação".

3 V. Ndou, "E-government for developing countries: opportunities and challenges"; R. C. G. Sant'ana, Mensuração da disponibilização de informações e do nível de interação dos ambientes informacionais digitais da administração municipal com a sociedade.

4 K. Frey et al., O acesso à informação. Caminhos da transparéncia: análise dos componentes de um sistema nacional de integridade. 
permite entender con mayor precisión las necesidades ciudadanas, facilitando la participación efectiva y directa en la gestión pública, y aumentando exponencialmente el valor de la información al compartirla abiertamente. ${ }^{5}$

Sin embargo, y como se verá más adelante, el acceso a la información gubernamental es parcial, superficial y complejo debido a variables tanto del contexto como del usuario y al volumen de datos públicos que no alcanza a ser dominado por los sistemas de integración y gerenciamiento existentes. ${ }^{6}$ La calidad del acceso a la información es crítica si se busca promover el desarrollo de puntos de vista independientes y relaciones transparentes entre Estado y sociedad, características claves en la participación ciudadana. ${ }^{7}$

Por otra parte, el empoderamiento ciudadano que los datos puedan generar está supeditado a garantizar su uso efectivo no sólo a través del acceso a la infraestructura informática sino también construyendo competencias informacionales, técnicas y éticas en los usuarios. ${ }^{8}$ De otra forma, el empoderamiento tecnológico refuerza la concentración de capacidades y el poder de aquellas minorías con el conocimiento, equipamento y contexto necesario para aprovechar las fuentes y flujos de datos existentes. ${ }^{\text {? }}$

Las problemáticas del empoderamiento informacional ciudadano en Brasil pueden ser claramente percibidas en el sector salud. Para entenderlas se deben estudiar tres áreas: a) la legislación y estructura del sector salud, b) las políticas públicas de amparo y estímulo al acceso a la información gubernamental, y c) las características de los sitios web suministrados por el Estado. A continuación comentamos cada una de estas tres áreas.

\section{EL CONTEXTO DE LOS SISTEMAS DE INFORMACIÓN PÚBLICA EN BRASIL Y EL SECTOR SALUD}

La constitución brasileña fundamentó el Sistema Único de Saúde (SUS) bajo un esquema descentralizado. Los estados y municipios tienen autonomía para administrar las acciones y recursos de la salud de acuerdo con sus necesidades particulares. ${ }^{10}$ El Fundo Nacional de Saúde (FNS) es el órgano encar-

Opengovdata, 8 Principles of Open Government Data.

J. Manyika et al., Big data: The next frontier for innovation, competition, and productivity.

R. C. G. Sant'ana y F. Rodrigues de Assis, "Acessando dados para visualização de afinidades nas votações entre parlamentares do Senado".

8 C. Berrío-Zapata, "Entre la Alfabetización Informacional y la Brecha Digital: reflexiones para una reconceptualización de los fenómenos de exclusión digital”.

9 M. B. Gurstein, "Open data: Empowering the empowered or effective data use for everyone?"; P. Norris, Digital divide: Civic engagement, information poverty, and the Internet world-

10 Ride. 
gado de distribuir y controlar la gestión de estos recursos. La participación ciudadana fue incluida en la gestión de salud por medio de la ley 8.142/90, con la conformación de colegios comunitarios denominados Conferências de Saúde y Conselhos de Saúde, cuya labor es fiscalizar la gestión correcta de tales recursos bajo los criterios establecidos por el decreto 1.232/94. ${ }^{11}$ Las transferencias se agrupan en bloques de financiamiento que se subdividen en componentes o bloques, que son grupos de acciones y programas a los cuales se deben asignar presupuestos específicos. Estos bloques son definidos en la Portaria 204/2007 para las áreas de a) atención básica, b) atención de complejidad ambulatoria y hospitalaria media o alta, c) vigilancia en salud, d) asistencia farmacéutica y e) gestión del SUS. ${ }^{12}$ Los profesionales que componen los colegios encargados de estos bloques deben tener conocimiento legal y burocrático del tema, y estar familiarizados con las necesidades de salud pública de su unidad territorial. Toda la información de esta estructura está compendiada en el sistema llamado DATASUS.

En 1988, la constitución brasileña reglamentó el acceso público a datos de la manera siguiente: "[...] todos têm direito a receber dos órgãos públicos informações de seu interesse particular, ou de interesse coletivo ou geral, que serão prestadas no prazo da lei, sob a pena de responsabilidade, ressalvadas aquelas cujo sigilo seja imprescindível à segurança da sociedade e do Estado" ${ }^{13}$

Este mismo documento instruyó sobre la creación de mecanismos de consulta de la información: "Cabem à administração pública, na forma da lei, a gestão da documentação governamental e as providências para franquear sua consulta a quantos dela necessitem". ${ }^{14}$

En el año 2011 se sancionó la Ley 12.527 de Acceso a la Información conocida como LAI, que adicionó nuevas obligaciones referentes al acceso público de la información y la confidencialidad en documentos gubernamentales. Organismos públicos y privados quedaron subordinados a esta ley, que modificó el orden legal anterior al convertir la "confidencialidad" de los documentos de gobierno de una característica general a una excepción par-

11 R. F. d. Brasil, Lei N ${ }^{\circ} 8.142$ de 28 de Dezembro de 1990. Dispõe sobre a participação da comunidade na gestão do Sistema Único de Saúde (SUS) e sobre as transferências intergovernamentais de recursos financeiros na área da saúde e dá outras providências; Decreto $\mathrm{N}^{\circ} 1.232$, de 30 de Agosto de 1994. Dispõe sobre as condições e a forma de repasse regular e automático de recursos do Fundo Nacional de Saúde para os fundos de saúde estaduais, municipais e do Distrito Federal, e dá outras providências

12 R. F. d. Brasil, Portaria N² 204 /GM de 29 de janeiro de 2007. Regulamenta o financiamento e a transferência dos recursos federais para as ações e os serviços de saúde, na forma de blocos de financiamento, com o respectivo monitoramento e controle.

13 Artículo 5o., inciso 33 de la Constituição da República Federativa do Brasil.

14 Idem. 
ticular. ${ }^{15}$ El uso de redes de datos para compartir documentos de gobierno se convirtió en una obligación: "Para cumprimento do disposto no caput, os órgãos e entidades públicas deverão utilizar todos os meios e instrumentos legítimos de que dispuserem, sendo obrigatória a divulgação em sítios oficiais da rede mundial de computadores (internet)".16

Ese año, Brasil se adhirió a la iniciativa multilateral Open Government Partnership, comprometiéndose a realizar esfuerzos por la transparencia y la efectividad en el acceso a informaciones. Se establecieron metas que serían periódicamente evaluadas por comités independientes, ${ }^{17}$ aun cuando Brasil ya había establecido políticas de inclusión digital con el decreto presidencial 6.991, de 2009, conocido como Plano Nacional de Inclusão Digital. ${ }^{18}$

\section{DATASUS, SU PROBLEMÁTICA Y EL CONCEPTO DE CAMPO DE INFORMACIÓN}

DATASUS es el sistema informático que compila y pone a disposición la información del SUS, sus acciones administrativas y su flujo financiero desde el gobierno federal, a través del Ministerio de Salud. Estos recursos se destinan, en términos del artículo 2 de la ley 8.142, de 1990, a los gastos del Ministerio, sus órganos y entidades de administración, así como a transferencias hacia los niveles estaduales y municipales de la nación. Su portal es conocido como DATASUS. La visibilidad de DATASUS fue paulatinamente creciendo así como la demanda que se hace de su información, y sin embargo, las relaciones entre el sistema y sus usuarios no mejoraron.

En el año de 2013, Rita Cassiano, de la Universidad Estadual Paulista UNESP, realizó un estudio de usuarios en la ciudad de Assis, São Paulo, Brasil ${ }^{19}$ para medir el nivel de dominio de DATASUS por parte de un grupo de profesionales del área de enfermería. Los resultados fueron preocupantes. Los usuarios, tanto trabajadores como estudiantes del sector salud, no consiguieron aprovechar la estructura de datos disponible por múltiples razones:

15 R. F. d. Brasil, Lei n 12.527 de 18 de novembro de 2011. Regula o acesso a informações previsto no inciso XXXIII do art. 5o, no inciso II do $\$ 30$ do art. 37 e no $\$ 20$ do art. 216 da Constituição Federal; altera a Lei no 8.112, de 11 de dezembro de 1990; revoga a Lei no 11.111, de 5 de maio de 2005, e dispositivos da Lei no 8.159, de 8 de janeiro de 1991; e dá outras providências.

16 Capítulo II, artículo 8o. de la Lei n 12.527 de 18 de novembro de 2011.

17 O. G. Partnership. Open Government Partnership Declaration.

18 R. F. d. Brasil, Decreto 6.991 de 27 de outoubro de 2009. Institui o Programa Nacional de Apoio à Inclusão Digital nas Comunidades - Telecentros.BR, no âmbito da política de inclusão digital do Governo Federal, e dá outras providências.

19 R. d. C. Cassiano Lopes, Percepção dos usuários sobre o processo de acesso a dados da saúde em sitios do Governo Federal. 
menús difíciles, hipervínculos rotos, desconocimiento sobre la mecánica de presentación de los datos e ignorancia sobre su estructura administrativa.

Lo preocupante es que esta situación no es novedosa ni en Brasil ni en el resto del mundo. ${ }^{20}$ Se pierde entre un tercio y la mitad del tiempo en línea por cuenta de interacciones desafortunadas con los sistemas de información. ${ }^{21}$ Se calcula que casi la mitad de los internautas de los EUA se sienten frustrados con la utilidad de los datos encontrados en los portales gubernamentales, su granularidad y la cantidad de tiempo invertida en sus búsquedas, esto debido a las terminologías utilizadas, su organización temática y a un soporte de metadatos deficiente. ${ }^{22}$

Otras explicaciones sobre las fuentes de frustración en los sitios web de gobierno han incluido factores como la baja predictibilidad de las interfaces, ${ }^{23}$ velocidad lenta de carga y descarga de las páginas, ${ }^{24}$ poca facilidad de visualización, dificultades de comprensión y soporte insuficiente en los portales. ${ }^{25}$ La situación se agrava si tomamos en consideración que estos sólo son los elementos "duros" de los sistemas de información, ${ }^{26}$ que representan sólo la fracción visible del problema. Bajo ellos están las propiedades sociotécnicas del sistema a las que Checkland llamó "blandas". Ellas configuran el "campo de información", 27 una estructura informacional tecnológica y sociocultural, transparente, histórica, contextual, individual y colectiva, que está cruzada por fuerzas en conflicto y cooperación, actuando bajo las leyes de la teoría de campo. ${ }^{28}$

20 V. S. Oliveira, Buscando interoperabilidade entre diferentes bases de dados: o caso da biblioteca do Instituto Fernandes Figueira; E. M. F. Barboza y E. M. d. A. Nunes, "A inteligibilidade dos websites governamentais brasileiros eo acesso para usuários com baixo nível de escolaridade".

21 J. Lazar et al., "Help! I'm lost: User frustration in web navigation".

22 K. Bessiere et al. Understanding Computer user frustration: Measuring and Modeling the disruption from poor designs.

23 B. Shneiderman, "Designing information-abundant web sites: issues and recommendations"; Designing The User Interface: Strategies for Effective Human-Computer Interaction, 4/e (New Edition).

$24 \mathrm{~J}$. Ramsay et al. "A psychological investigation of long retrieval times on the World Wide Web."

25 Bessiere et al., Understanding Computer user frustration...

26 P. Checkland, "Systems thinking."

27 Berrío-Zapata, "Una visión crítica de la intervención en Tecnologías de la Información y Comunicación (TIC) para atacar la brecha digital y generar desarrollo sostenible en comunidades carenciadas en Colombia: el proyecto Cumaribo."

28 K. Lewin, La teoría del campo en la ciencia social. 
El "campo de información" se puede definir desde cinco perspectivas:

a) Una arquitectura técnica y tecnológica que incluye estructuras de micropoder, vigilancia y control, que guían la recuperación de la información y monitorean a los usuarios dentro de un espacio de acción normalizador actuando como un Panopticon Digital. ${ }^{29}$

b) Una estructura semiótica, ideológica y cultural de isotopías que trasladan e implantan representaciones y rutinas de una sociedad a otra. ${ }^{30}$ Su medio de transmisión principal son las nomenclaturas clasificatorias. ${ }^{31}$

c) Un campo de conflicto y cooperación entre los agentes informacionales en términos de Negociación Simbólica ${ }^{32}$ y Negociación de Significado. ${ }^{33}$

d) Un espacio de acción del comportamiento informacional de los usuarios y formuladores del sistema.

e) Un objeto epistemológico, histórico, cultural e ideológicamente considerado, estudiado y explicado desde el sentido común, la ciencia y la tecnología. ${ }^{34}$

Estas características deberían de ser parte de las preocupaciones de quienes diseñan políticas de información, pues representan el acervo de más de 50 años investigación en ciencias sociales para una comprensión más depurada de los fenómenos informacionales y, con ello, la posibilidad de mayor eficiencia en el desarrollo sus políticas relacionadas. Sin embargo, la conceptualización de políticas públicas de información aún carece de abordaje interdisciplinar y visión de complejidad. Y así, las consecuencias de estas falencias terminan apareciendo en sistemas de información críticos como DATASUS.

29 S. Zuboff, "Be the friction: Our Response to the New Lords of the Rings."

30 I. Blikstein, Kaspar Hauser ou a fabricação da realidade; C. Avgerou, "Information systems in developing countries: a critical research review."

$31 \mathrm{H}$. A. Olson, The power to name: locating the limits of subject representation in libraries.

32 En el campo de la informática Küngas define la negociación simbólica, como un proceso donde las partes tratan de llegar a un acuerdo sobre los medios intelectivos para el logro de sus objetivos, mediante la aplicación de técnicas de razonamiento simbólico. Esta es una definición tendiente al debate lógico matemático incluyendo elementos socioculturales: la negociación y lo simbólico. P. Küngas y M. Matskin. "Partial deduction for linear logic: the symbolic negotiation perspective".

33 La negociación de significado para Bouquet es cualquier enfoque viable para la interoperabilidad semántica entre entidades autónomas, que no pueden evaluar problemas semánticos "mirando dentro de la cabeza del otro", así que forzosamente aceptan un proceso social de negociación y acuerdos sobre el significado (semántica) y la intención del hablante (pragmática) en su proceso de comunicación. Burato los define sencillamente como el proceso general con el que los agentes llegan a un acuerdo sobre el significado de un conjunto de términos. P. Bouquet y M. Warglien. Meaning negotiation: an invitation; E. Burato et al. "Meaning Negotiation as Inference."

34 K. Tuominen et al. "Information Literacy as a Sociotechnical Practice." 


\section{LA CIENCIA DE LA INFORMACIÓN COMO MOVILIZADORA DE LA GESTIÓN DE POLÍTICAS PÚBLICAS INFORMACIONALES}

¿Por qué los fenómenos informacionales son desatendidos en el campo de la gestión de políticas públicas? ¿Por qué vemos repetirse casos como el de DATASUS en la esfera informacional pública regional? La ciencia política latinoamericana recorre un camino en el que coexisten visiones deterministas y de complejidad, ${ }^{35}$ pero en la práctica, se cae en procesos formalistas que acaban con cualquier visión dialéctica o participativa. Existe una tradición autoritaria, institucionalizada en los sistemas de acción política a través de lo que O’Donnell llamó el "Estado burocrático autoritario". ${ }^{36}$

Todo análisis sobre formulación de políticas informacionales está condicionado por el contexto, la deliberación pública y la construcción de discursos ${ }^{37}$ factores afectados por la tendencia a sobresimplificar y, con ello, partícipes de la pérdida de parte significativa de los aspectos esenciales que determinan las políticas públicas. ${ }^{38}$ Por esta razón, la perspectiva interdisciplinar de la ciencia de la información (CI) resulta muy interesante para movilizar la formulación de políticas públicas de información.

Para iniciar este acercamiento entre la CI y las políticas de información, se elaboran a continuación varias reflexiones utilizando la matriz de análisis de políticas de información de Moore, ${ }^{39}$ en las dimensiones de "recursos humanos" y "mercados de políticas de información" propuestas por Sebastián y Rodríguez. ${ }^{40}$ Para guiar esta discusión se van a proponer dos cuestiones centrales:

1) La legitimación y ajuste de las arquitecturas informacionales basadas en Internet.

2) La construcción de "empatía" entre las estructuras informacionales y las necesidades, sentires y capacidades de sus usuarios.

35 G. Flexor y S. P. Leite. "Análise de políticas públicas: breves considerações teórico-metodológicas."

36 G. A. O'Donnell, Modernization and bureaucratic-authoritarianism: Studies in South American politics; "Reflections on the patterns of change in the bureaucratic-authoritarian state."; Catacumbas.

37 S. H. Linder y B. G. Peters. "A metatheoric analysis of policy design."

38 Flexor y Leite, "Análise de políticas públicas...".

39 N. Moore, Information policy and strategic development: a framework for the analysis of policy objectives.

40 M. C. Sebastián et al. "La necesidad de políticas de información ante la nueva sociedad globalizada." 
En esta discusión, el Estado tiene un rol protagónico en términos de negociación y violencia simbólica ${ }^{41}$ (negociar e imponer representaciones sociales por decreto o cooptación). Por ello sus agentes deben tener ideas claras y amplias sobre los costos de ajuste e implantación de rutinas informacionales nuevas, y la deconstrucción simbólica del mercado informacional que esto supone. Para esos efectos, las teorías que se discuten a continuación contribuyen con conceptos centrales.

\section{EL CONTEXTO Y EL USUARIO SON CRÍTICOS}

A la ciencia de la información le tomó 40 años llegar a comprender la importancia del contexto en el cual se encuentran los sistemas de información. Al igual que la ciencia informática, la ciencia de la información nació durante la caída de las potencias europeas y el surgimiento de EUA y la URSS; un periodo de reformulación del proyecto científico, industrial y capitalista. Inicialmente, ambas eran ciencias prácticas, de perspectiva hermenéutica y crítica nula ${ }^{42}$ con una visión de totalidad monumentalista heredada del pensamiento eurocéntrico de Paul Otlet. ${ }^{43}$ Consideraciones sobre "el usuario" o contextos distintos al europeo eran inexistentes. Finalizando el siglo XX, al popularizarse la informática, la cuestión sobre el uso ciudadano de la información y sus públicos ganó importancia. ${ }^{44}$ Después, en 1976, Brenda Dervin atacó los presupuestos que esgrimían los "expertos" sobre las necesidades de los usuarios. ${ }^{45}$ Veinte años después apareció la epistemología construccionista social en la ciencia de la información, ${ }^{46}$ se profundizó sobre el papel del contexto $^{47}$ y se articularon las perspectivas ecológica y evolucionista al campo disciplinar de la ciencia de la información. ${ }^{48}$

Toda esta serie de evoluciones, aplicadas al diseño de políticas de información, significan considerar profundamente las condiciones históricas, geo-

41 P. Bourdieu, "Structures, habitus, power: Basis for a theory of symbolic power"; M. N. Gonzalez de Gomez, "Novos cenários políticos para a informação"; Bourdieu, "La fabrique de l'habitus économique"; C. A. Tamayo Gómez et al. "Génesis del campo de Internet en Colombia: elaboración estatal de las relaciones informacionales".

42 L. J. McCrank, Historical information science: An emerging unidiscipline.

43 I. Rieusset-Lemarié, "P. Otlet's mundaneum and the international perspective in the history of documentation and information science."

44 B. M. Wildemuth y D. O. Case. "Early information behavior research."

45 B. Dervin, "Strategies for dealing with human information needs: Information or communication?"

46 K. Tuominen y H. Savolainen. A Social Constructionist Approach to the Study of Information Use as Discursive Action; Tuominen et al., "Information Literacy...".

47 C. Courtright, "Context in information behavior research."

48 A. Spink y J. Currier. "Emerging Evolutionary Approach to Human Information Behavior". 
gráficas y culturales de la población objetivo así como aplicar una hermenéutica interdisciplinar para caracterizar los nichos y perfiles de los usuarios que serán sujeto de la gobernanza informacional propuesta.

\section{COMPORTAMIENTO INFORMACIONAL EN USUARIOS Y FORMULADORES}

El rol del usuario y del formulador de un sistema de información no es fijo; cambia y se alterna en la medida en que la actividad del sujeto y su contexto van cambiando. Lo que persiste es el egocentrismo de los sujetos implicados. Las políticas y sistemas informacionales se formulan desde suposiciones e intereses de grupo. Aun con las mejores intenciones, un sistema de información encarna una fuerza invasora desde el punto de vista de los usuarios. Es necesaria una negociación simbólica eficiente para lograr efectos visibles y duraderos, así como monitorear los procesos de autoorganización que van paulatinamente emergiendo de los usuarios. Estos elementos emergentes son intrincados e imprevistos, así que deben ser asumidos bajo una visión estratégica, de complejidad y caos. ${ }^{49}$ El problema central es el sentido de las acciones y las estructuras para los agentes participantes. ${ }^{50}$

La relación entre los diseñadores de políticas y los usuarios no es un espacio vacío, sino que en él diversos grupos de interés juegan sus cartas colaborativa o conflictivamente, de forma abierta o disimulada. Cada facción lucha por su oportunidad de incrementar los grados de libertad de sus decisiones hasta límites que dependen de la fortaleza institucional del contexto. ${ }^{51}$ Aquí el problema central son las asimetrías de información y la construcción de formas para balancearlas de manera autoregulada y sostenible.

\section{RACIONALIDAD, EL SENTIDO DE LA INFORMACIÓN, INFORMÍVOROS Y COMPORTAMIENTO DE ENJAMBRE}

El comportamiento informacional no puede ser asumido como "racional", algo que ya había sido establecido por la teoría de la racionalidad limitada. ${ }^{52}$ Sabemos por Brenda Dervin que a) dar más información no es necesaria-

49 K. M. Eisenhardt y S. L. Brown. "Competing on the edge: strategy as structured chaos."

50 Dervin, "Sense-making theory and practice: an overview of user interests in knowledge seeking and use."

51 M. Crozier, La sociedad bloqueada; P. Medellín Torres, La política de las políticas públicas: propuesta teórica y metodológica para el estudio de las políticas públicas en países de frágil institucionalidad.

52 H. A. Simon, Models of bounded rationality. 
mente mejor; b) que no se consume información fuera de contexto; c) que los canales de información formales no siempre son los mejores, y d) que las múltiples funciones de la información no son simples, específicas o pragmáticas. El objeto de la información es crear sentido, conceptualizar; el saber es más un verbo que un sustantivo, es diversidad y complejidad. El comportamiento informacional, como acción cotidiana humana mediada por el lenguaje, es una construcción social y se edifica a través de negociaciones de significado. El diseño de políticas públicas debe tener en cuenta la cotidianidad del usuario, apoyado en el concepto de tejido social. ${ }^{53}$ Hay que entender los flujos de información en la lógica comunitaria, desde los "pequeños mundos" ${ }^{24}$ y sus estructuras de gratificación social. ${ }^{55}$ Las políticas de información deben ser diseñadas con una visión bottom-up, desde la comunidad y no desde la técnica, la moda tecnológica o el imaginario de los formuladores. Deben existir ruedas consultivas con los usuarios y grupos de interés, así como negociación y espacio para ajustes permanentes.

El ser humano es un informívoro, ${ }^{56}$ un rumiante de información que rehúye la disonancia cognitiva y la incertidumbre. Es un cazador de datos que procura construir sentido de formas energéticamente económicas. La credibilidad se antepone a la exactitud, la conveniencia sobre la veracidad, la consonancia sobre la disonancia. Una arquitectura informacional no es aceptada tanto por su excelencia técnica como por el sentido que tenga para el usuario y su economía energética. Aunque vivamos un mundo grafocéntrico, ${ }^{57}$ los canales formales escritos no necesariamente son los más atendidos. Y si la información es adobada con elementos lúdicos, con certeza tendrá una mejor aceptación. $^{58}$

Los seres humanos actúan en enjambre, ${ }^{59}$ estructuras de encadenamientos informacionales que impulsan de forma inconsciente la acción colectiva coordinada, movilizando mecanismos ancestrales que se apoyan en lo emotivo $^{60}$ y en el comportamiento de manada. Esta es una característica que no puede ser dejada de lado al diseñar políticas de información.

53 Tuominen y Savolainen. A Social Constructionist Approach...

54 Concepto de Efreda Chatman que refiere al poder que un grupo social pequeño y cerrado ejerce sobre la actividad informacional de sus miembros.

55 E. A. Chatman, "Life in a small world: Applicability of gratification theory to information-seeking behavior."; "The impoverished life-world of outsiders."

56 G. A. Miller, "Informavores".

57 H. M. Serres, Hominescências: O começo de uma outra bumanidade.

58 W. Stephenson, The play theory of mass communication.

59 S. Gutiérrez et al. "Swarm Intelligence Applications for the Internet."

60 D. Nahl, "The Centrality of the Afective in Information Behavior". 


\section{ASIMETRÍAS DE INFORMACIÓN, ECONOMÍA ENERGÉTICA Y OPORTUNISMO}

Ronald Coase percibió que toda transacción económica es informacional e involucra costos: de búsqueda, negociación y salvaguarda. ${ }^{61}$ La transacción es una estructura de intercambio de información que estipula contraprestaciones futuras con respecto a la propiedad de un bien o servicio cualquiera, y cláusulas de protección a lo prometido. Nunca accederemos a toda la información porque los costos de búsqueda serían inviables. Actuando con información limitada, preferimos datos de fácil acceso independiente de su calidad; intercambiamos información con agentes que nos sean familiares aunque no sean idóneos; preferimos negociaciones simplificadas así sean menos favorables. Este comportamiento de mínimo esfuerzo informacional fue observado en la lingüística, ${ }^{62}$ la bibliotecología ${ }^{63}$ y la ciencia informática. ${ }^{64}$

El costo de transacción y la tendencia al mínimo esfuerzo son fuentes de asimetría informacional. Los agentes con mayor capacidad económica/ energética podrán acceder a más y mejor información ganando ventajas. Miembros de gobierno, representantes organizacionales y trabajadores de conocimiento son ejemplos de colectivos con capacidad para sufragar altos costos de información, incluyendo barreras técnicas y de competencia informacional. Esta concentración de poder tenderá a institucionalizarse, naturalizándose como "orden debido". Una vez naturalizada, los grupos de interés dominantes tenderán a rechazar cambios tecnológicos o políticos que hagan incierto su dominio y arriesguen su capital informacional. Toda política tendrá que ofrecer, mercadear y forzar simultáneamente sus propuestas para mudar el estado de las cosas.

Esta situación de asimetría esta cruzada por lo que Williamson definió como "oportunismo", la tendencia de los agentes económicos a tomar ventaja de sus contrapartes durante las transacciones, indiferentes a los perjuicios que pudiesen causar. ${ }^{65}$ El oportunismo es la base del dilema agente-principal de la teoría de la agencia. ${ }^{66}$ Un actor social que llamaremos "principal" (puede ser individual o colectivo) escoge y designa formalmente a un "agente" para que actúe en su representación. El agente, una vez tomada posesión de su calidad de representante con poderes, manipula los flujos de información

61 R. Coase, The firm, the market and the law.

62 G. K. Zipf, Human behavior and the principle of least effort: an introduction to buman ecology.

63 Z. Liu y Z. Y. L. Yang. "Factors influencing distance-education graduate students' use of information sources: A user study."

64 C. N. Mooers, "Mooers' Law or Why Some Retrieval Systems Are Used and Others Are Not.”; L. A. Adamic y B. A. Huberman. "Zip's law and the Internet."

65 O. Williamson y S. E. Masten, The economics of transaction costs.

66 S. A. Ross, "The economic theory of agency: The principal's problem"; B. Mitnick, "Origin of the theory of agency: an account by one of the theory's originators". 
que recibe a nombre de su representado para incrementar su poder y salvaguardar sus intereses. Los mandatarios de elección popular y los burócratas que actúan en representación de los ciudadanos están afectados por el fenómeno de la agencia y, por tanto, su gestión de las políticas de información se verá influida por intereses minoritarios y maniobras tendientes a mantener el monopolio de información que ejercen. ${ }^{67}$ Toda política informacional acarreará cambios en las relaciones de agencia estatuidas entre gobierno, ciudadanos y estado, creando incertidumbre y oposición entre los cuerpos políticos y de gobierno que se vean afectados, causando posibles boicots encubiertos. El comportamiento de los gestores de gobierno también debe tener seguimiento y ser sujeto de ajustes permanentes. Toda política es un proceso de aprendizaje y reacomodo perenne.

Como la información es limitada, costosa de obtener y sujeta a intereses oportunistas, aquélla considerada óptima y estratégica tenderá a ser retenida por grupos de interés poderosos. Aplicando el teorema de los limones de Akerlof, ${ }^{68}$ se deduce que frente a la monopolización de la mejor información y las dificultades del ciudadano común para diferenciar la información de "mala calidad", el consumo de esta última tenderá a crecer. ¿Pero, quién define lo que es "buena información"? Este es el problema de la "relevancia de la información", que ha sido central para Saracevic, ${ }^{69}$ quien concluyó que lamentablemente no hemos invertido recursos en investigar este problema, aun cuando con la globalización y popularización de los medios informáticos se tornó un problema público. Con la privatización de los medios de búsqueda y recuperación el problema de la relevancia se "privatizó" y los estudios sobre relevancia poco se han traducido en mejoras de los sistemas de información.

En conclusión, entender la información en términos de sus costos de transacción, en un contexto de asimetrías, agencia, oportunismo y monopolio, es necesario para diseñar políticas realistas. También es necesario investigar el concepto de relevancia que encarna cada política, cada grupo de usuarios, cada circunstancia, pero lamentablemente hoy, esa área de investigación ha sido descuidada o dejada al albedrío de las grandes corporaciones de la información.

67 Eisenhardt, "Agency theory: An assessment and review".

68 G. A. Akerlof, "The market for" lemons": Quality uncertainty and the market mechanism".

69 Saracevic, T. "The concept of "relevance" in information science: A historical review"; "Relevance: A review of and a framework for the thinking on the notion in information science."; "Relevance: A review of the literature and a framework for thinking on the notion in information science. Part III: Behavior and effects of relevance". 


\section{COMPETENCIAS INFORMACIONALES}

DEL CIUDADANO Y EMPODERAMIENTO

Un último aporte al estudio de políticas de información es el concepto de I-Literacy o alfabetización informacional (ALFIN). El desarrollo de competencias informacionales requiere de alfabetismo en tecnologías informáticas y lectoescritura, pero éstas son condiciones necesarias mas no suficientes para la ALFIN. Se requieren dos niveles más: el nivel de metaanálisis o capacidad para evaluar y replicar fuentes, y la habilidad crítica comprensiva que permita comprender discursos y proponer alternativas. Es una cadena de procesamiento analítico, sintético y compartido de información para debatir y generar propuestas nuevas. ${ }^{70} \mathrm{El}$ último nivel configura lo que Capurro denomina la "acción moral responsable", ${ }^{71}$ aquélla fundamentada e informada que exige comunicación con otros agentes en diálogo crítico permanente para obtener datos, reflexionar en conjunto y mantener disposición para modificar opiniones.

Crear competencias informacionales es difícil y caro de gestionar porque implica cambios profundos en materia de educación y empoderamiento. Requiere asegurar derechos fundamentales como la educación y la libertad de expresión. Significa pensar en términos amplios sobre variables aparentemente ajenas a las políticas de información, pero que determinan su éxito o fracaso.

\section{Conclusiones}

Los países "periféricos" se matricularon tardíamente en el orden informacional digital, y por su falta de experiencia y capital humano, terminaron reforzando su relación de dependencia con Europa y Norteamérica e importando políticas de moda de forma irreflexiva ni conocimiento de causa. Estas políticas tienden a chocar con las características típicas del orden informacional de los países en desarrollo, una realidad poco investigada y por tanto desconocida. La gestión de políticas de la información en Latinoamérica nació plena de problemas de dependencia, restricción, inequidad y autoritarismo.

Es urgente un abordaje diferente, que puede ser construido a partir de las ciencias sociales y la investigación en ciencia de la información con respecto al comportamiento informacional humano. Este trabajo compiló varias de sus teorías para contrastarlas con un caso real como el de DATASUS,

70 Berrío-Zapata, "Entre la Alfabetización Informacional...”.

71 R. Capurro, "Información y acción moral en el contexto de las nuevas tecnologías". 
ejercicio que presentamos en la Figura 1. Este ejercicio permitió construir un diagrama del "campo informacional" de la salud en Brasil, en cuyo centro está DATASUS. Esta visión de conjunto permite articular múltiples fenómenos y líneas de investigación que podrían dar fundamentación empírico-teórica a los formuladores de políticas de información desde una visión resumida mas no simplificada.

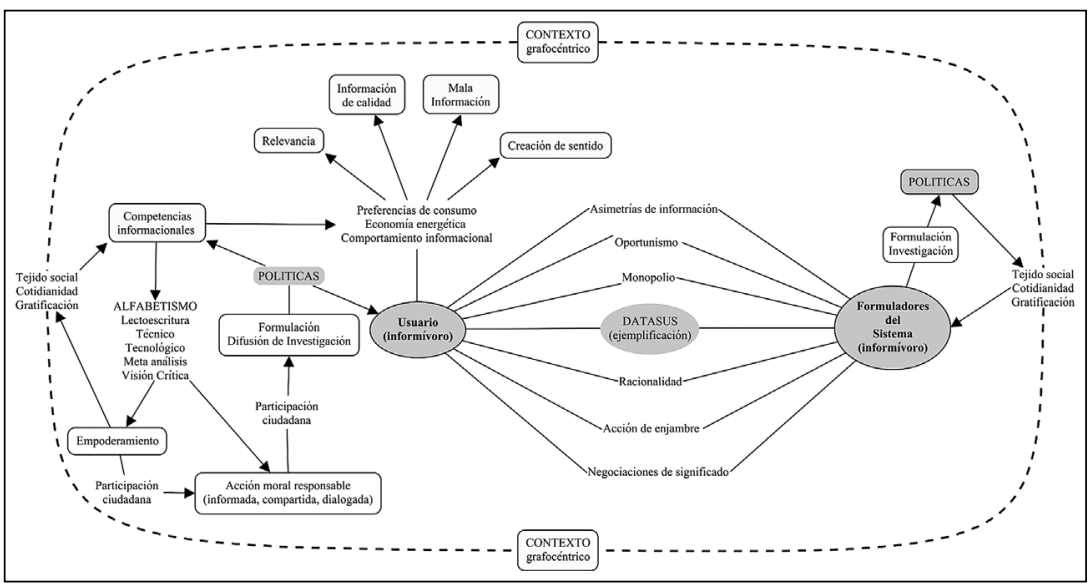

Figura 1. Diagrama del "campo de información" estructurado por los factores que afectan al desarrollo de políticas de información, ejemplificado con el sistema de información de salud del Brasil, DATASUS. Cada nodo corresponde a los diferentes conceptos teóricos desarrollados en este artículo y es un área de investigación a ser desarrollada. Fuente: elaboración propia.

De cada área teórica tratada en este artículo se generan diversas preguntas de investigación, que pueden ser:

- Contexto: ¿cuál es el contexto de los usuarios de DATASUS? ¿Son mayoritariamente urbanos? ¿Están en ciudades pequeñas? ¿Cuál es la historia de DATASUS y cuáles son sus stakeholders? ¿Qué intereses y conflictos alinean a estos stakeholders?

- Sentido: ¿cómo perciben, representan y asocian los diversos usuarios y stakeholders a DATASUS? ¿Estas representaciones están asociadas con otros procesos o características sociales y culturales? ¿Cuál es la definición y nivel de relevancia del sistema y sus contenidos desde la perspectiva de los usuarios y los stakeholders? ¿Cuál es el grado de "intuitividad" del sistema? ¿Cuáles son sus elementos lúdicos y de gratificación? ¿Cuál es la racionalidad del sistema y cuál la del usuario? ¿Existen espacios de "negociación simbólica" con los usuarios? 
- Asimetrías: ¿cuáles son los actores del sistema que pueden ser sujetos de fenómenos de agencia, oportunismo, monopolio de información y que asimetrías podrían causar? ¿Cómo se pueden contrarrestar de forma sostenible y autoregulada estas desigualdades?

- Energético: ¿cuál es el grado de usabilidad del sistema en términos de esfuerzo? ¿Cuál es el nivel de complejidad de sus contenidos y representaciones de datos?

- ALFIN: ¿cuál es el nivel de ALFIN en los usuarios en los diferentes campos que convergen en el sistema (legal, médico, burocrático, etc.)? ¿Está alineado el sistema con las competencias de los "usuarios objetivo"?

- Control: ¿qué tipo de fenómenos de "enjambre" puede desatar el sistema? ¿Qué tipo de consecuencias pueden tener esos fenómenos? ¿Cómo anticipar y controlar las consecuencias negativas?

Estas áreas de discusión se pueden multiplicar en un ejercicio completo de revisión o evaluación de cualquier proyecto de política pública de información. La utilidad de este tipo de cuestionamientos y estructuras de pensamiento radica en intentar preservar la complejidad implicada en el desarrollo de políticas, manteniendo una estructura organizada y sistemática basada en modelos teóricos consistentes. Este ejercicio también permite pensar perspectivas hasta ahora poco exploradas, como aquellas relativas a los problemas de poder como la agencia, el monopolio de información y el oportunismo, o aplicar el principio del "menor esfuerzo".

En el proyecto de la sociedad de la información coexisten el sueño de una red pública de datos libres y gratuitos con la pesadilla de la vigilancia, el control, la colonización electrónica y otras formas de concentración de poder. Los actores dominantes esperan perpetuar las asimetrías de información que hasta ahora les reportan ventajas, y sólo aquellas políticas de estado conscientes de este tipo de conflictos podrán balancear y controlar tales efectos. Sin embargo, los gestores de políticas terminan actuando bajo esquemas autoritarios que no interrogan la realidad local ni crean espacios de negociación con los usuarios. Copian perspectivas importadas y las imponen por fuerza de ley, sustentándolas con consignas demagógicas. La inversión de recursos se centra en las cosas y descuida a las personas. Sin investigación ni usuarios alfabetizados técnica y éticamente, los avances tecnológicos se seguirán convirtiendo en asimetrías informacionales mayores. La población se hará más manipulable, debilitando el ya endeble orden institucional. Los avances normativos serán cosméticos y así, todo seguirá cambiando, pero al final, nada se modificará. 


\section{BiBLIOGRAFÍA}

Adamic, L. A. y B. A. Huberman. "Zip's law and the Internet." Glottometrics 3 (1) (2000): 143-150.

Akerlof, G. A. "The market for" lemons": Quality uncertainty and the market mechanism." The quarterly journal of economics (1970): 488-500.

Avgerou, C. "Information systems in developing countries: a critical research review." Journal of Information Technology 23 (3) (2008): 133-146.

Barboza, E. M. F. y E. M. d. A. Nunes. "A inteligibilidade dos websites governamentais brasileiros eo acesso para usuários com baixo nível de escolaridade.” Inclusão Social 2 (2) (2007): 19-33.

Berrío-Zapata, C. "Una visión crítica de la intervención en Tecnologías de la Información y Comunicación (TIC) para atacar la brecha digital y generar desarrollo sostenible en comunidades carenciadas en Colombia: el proyecto Cumaribo." Management XIV (23-24) (2005): 165-181.

"Entre la Alfabetización Informacional y la Brecha Digital: reflexiones para una reconceptualización de los fenómenos de exclusión digital." Revista Interamericana de Bibliotecología 35 (1) (2012): 39-53.

Bessiere, K. et al. Understanding Computer user frustration: Measuring and Modeling the disruption from poor designs. Digital Repository at the University of Maryland. Maryland, University of Maryland Computer Science Department. CS-TR-4409 UMIACS; UMIACS-TR-2002-89 HCIL-TR-2002-18, 2003.

Blikstein, I. Kaspar Hauser ou a fabricação da realidade. São Paulo: Editora Pensamento-Cultrix Ltda, 2003.

Bouquet, P. y M. Warglien. Meaning negotiation: an invitation. AAAI Workshop 2002 Edmonton, Association for the Advancement of Artificial Intelligence (AAAI), 2002.

Bourdieu, P. "Structures, habitus, power: Basis for a theory of symbolic power”, en N. B. Dirks, G. Eley y S. B. Ortner (ed.), Culture/ power/history: A reader in contemporary social theory. New Jersey, Princenton University Press: 155-199, 1994.

- "La fabrique de l'habitus économique." Actes de la recherche en sciences sociales (5) (2003): 79-90.

Brasil, R. F. d. Constituição da República Federativa do Brasil. Brasilia, 1988.

Brasil, R. F. d. Lei No 8.142 de 28 de Dezembro de 1990. Dispõe sobre a participação da comunidade na gestão do Sistema Único de Saúde (SUS) e sobre as transferências intergovernamentais de recursos financeiros na área da saúde e dá outras providências. Brasilia, 1990. 
Brasil, R. F. d. Decreto No 1.232, de 30 de Agosto de 1994. Dispõe sobre as condições e a forma de repasse regular e automático de recursos do Fundo Nacional de Saúde para os fundos de saúde estaduais, municipais e do Distrito Federal, e dá outras providências. Brasilia, 1994.

Brasil, R. F. d. Portaria No 204 /GM de 29 de janeiro de 2007. Regulamenta o financiamento e a transferência dos recursos federais para as ações e os serviços de saúde, na forma de blocos de financiamento, com o respectivo monitoramento e controle. Brasilia, 2007.

Brasil, R. F. d. Decreto 6.991 de 27 de outoubro de 2009. Institui o Programa Nacional de Apoio à Inclusão Digital nas Comunidades - Telecentros.BR, no âmbito da política de inclusão digital do Governo Federal, e dá outras providências. Brasilia, 2009.

Brasil, R. F. d. Lei $n^{\circ} 12.527$ de 18 de novembro de 2011. Regula o acesso a informações previsto no inciso XXXIII do art. 5o, no inciso II do $\$ 3$ o do art. 37 e no $\$ 20$ do art. 216 da Constituição Federal; altera a Lei no 8.112, de 11 de dezembro de 1990; revoga a Lei no 11.111, de 5 de maio de 2005, e dispositivos da Lei no 8.159, de 8 de janeiro de 1991; e dá outras providências. Brasilia, 2011.

Brasil, R. F. d. Lei n 12.527 de 18 de novembro de 2011. Regula o acesso a informações previsto no inciso XXXIII do art. 5o, no inciso II do $\$ 3$ o do art. 37 e no $\$ 2$ o do art. 216 da Constituição Federal; altera a Lei no 8.112, de 11 de dezembro de 1990; revoga a Lei no 11.111, de 5 de maio de 2005, e dispositivos da Lei no 8.159, de 8 de janeiro de 1991; e dá outras providências. Capítulo II, Artículo $8^{\circ}$, inciso 2. Brasilia, 2011.

Burato, E. et al. "Meaning Negotiation as Inference." Arxiv.org Cornell University. Ithaca NY, Cornell University. arXiv:1101.4356v1 (2011): 1-54.

Capurro, R. "Información y acción moral en el contexto de las nuevas tecnologías." VII Encontro Internacional de Informação, Conbecimento, Ética e Ação, Marília, Brasil, Universidade Estadual Paulista (UNESP), 2011.

Cassiano Lopes, R. d. C. Percepção dos usuários sobre o processo de acesso a dados da saúde em sítios do Governo Federal. Encontro Internacional Dados, Tecnologia e Informação. Marilia, Universidade Estadual Paulista UNESP, 2013.

Chatman, E. A. "Life in a small world: Applicability of gratification theory to information-seeking behavior." Journal of the American Society for information science 42 (6) (1991): 438-449.

- "The impoverished life-world of outsiders." Journal of the American Society for information science 47 (3) (1996): 193-206.

Checkland, P. "Systems thinking." Rethinking management information systems (1999): 45-56.

Coase, R. The firm, the market and the law. Chicago: University of Chicago, 1988. 
Courtright, C. "Context in information behavior research." Annual Review of Information Science and Technology 41 (1) (2007): 273 306.

Crozier, M. La sociedad bloqueada. Buenos Aires, Amorrortu, 1970.

Dervin, B. "Strategies for dealing with human information needs: Information or communication?" Journal of Broadcasting E Electronic Media 20 (3) (1976): 323-333.

"Sense-making theory and practice: an overview of user interests in knowledge seeking and use." Journal of knowledge management 2 (2) (1998): 36-46.

Eisenhardt, K. M. "Agency theory: An assessment and review." Academy of management review 14 (1) (1989): 57-74.

Eisenhardt, K. M. y S. L. Brown. "Competing on the edge: strategy as structured chaos.” Long Range Planning 31 (5) (1998): 786-789.

Flexor, G. y S. P. Leite. "Análise de políticas públicas: breves considerações teórico-metodológicas." Encontro Nacional de Economia Política 12 (2007).

Frey, K. et al. O acesso à informação. Caminhos da transparéncia: análise dos componentes de um sistema nacional de integridade. B. W. Speck. Campinas, Editora da Unicamp, 2002.

Gonzalez de Gomez, M. N. "Novos cenários políticos para a informação." Ciência da Informação 31 (1) (2002): 27-40.

Gurstein, M. B. "Open data: Empowering the empowered or effective data use for everyone?" First Monday 16 (2) (2011).

Gutiérrez, S. et al. "Swarm Intelligence Applications for the Internet." Encyclopedia of Internet Technologies and Applications. Hershey, PA: Information Science Publishing, 2007.

Küngas, P. y M. Matskin. "Partial deduction for linear logic: the symbolic negotiation perspective”, en J. Leite, A. Omicini, P. Torroni y P. Yolum, DALT 2004: Declarative Agent Languages and Technologies. Berlin, Springer-Verlag. LNAI 3476: 35-52, 2005.

Lazar, J. et al. "Help! I'm lost: User frustration in web navigation." It ESociety 1 (3) (2003): 18-26.

Lewin, K. La teoría del campo en la ciencia social. Buenos Aires, Editorial Paidós, 1978.

Linder, S. H. y B. G. Peters. "A metatheoric analysis of policy design", en W. N. Dunn y R. M. Kelly, Advances in policy studies since 1950. London, Transaction Publishers, vol. 10: 201-237, 1992.

Liu, Z. y Z. Y. L. Yang. "Factors influencing distance-education graduate students' use of information sources: A user study." The Journal of Academic Librarianship 30 (1) (2004): 24-35.

Malin, A. M. B. "Gestão da informação governamental: em direção a uma metodologia de avaliação." DataGramaZero - Revista de Ciência da Informação 7 (5) (2006).

Manyika, J. et al. Big data: The next frontier for innovation, competition, and productivity. New York: McKinsey Global Institute, 2011. 
McCrank, L. J. Historical information science: An emerging unidiscipline, Information Today, Inc., 2001.

Medellín Torres, P. La política de las políticas públicas: propuesta teórica y metodológica para el estudio de las políticas públicas en países de frágil institucionalidad. Proyecto "Política y políticas públicas en los procesos de reformas en América Latina. Similitudes y Diversidades" (Proyecto FRA/02/073). Santiago de Chile, Naciones Unidas, CEPAL, 2004.

Miller, G. A. "Informavores", en F. Machlup y U. Mansfield, The study of information: Interdisciplinary messages. New York, Wiley-Interscience: 111-113, 1983.

Mitnick, B. "Origin of the theory of agency: an account by one of the theory's originators.” Available at SSRN 1020378, 2006.

Mooers, C. N. "Mooers' Law or Why Some Retrieval Systems Are Used and Others Are Not." Bulletin of the American Society for Information Science and Technology 23 (1) (1996): 22-23.

Moore, N. Information policy and strategic development: a framework for the analysis of policy objectives. Aslib proceedings, MCB UP Ltd., 1993.

Nahl, D. "The Centrality of the Afective in Information Behavior", en D. Nahl y D. Bilal, Information and emotion: The emergent affective paradigm in information behavior research and theory. Medford, NJ, Information Today Inc: 3-37, 2007.

Ndou, V. "E-government for developing countries: opportunities and challenges." The Electronic Journal of Information Systems in Developing Countries 18 (2004).

Norris, P. Digital divide: Civic engagement, information poverty, and the Internet worldwide. New York, Cambridge University Press, 2001.

O'Donnell, G. A. "Reflections on the patterns of change in the bureaucratic-authoritarian state." Latin American Research Review 13 (1) (1978): 3-38.

Modernization and bureaucratic-authoritarianism: Studies in South American politics. Institute of International Studies, University of California Berkeley, 1973.

_ Catacumbas. Buenos Aires, Prometeo Libros Editorial, 2008.

O. G. Partnership. Open Government Partnership declaration, 2011. http://www.opengovpartnership.org/about

Oliveira, V. S. Buscando interoperabilidade entre diferentes bases de dados: o caso da biblioteca do Instituto Fernandes Figueira. Fundação Instituto Oswaldo Cruz Rio de Janeiro Escola Nacional de Saúde Pública Sergio Arouca, 2005. Mestre.

Olson, H. A. The power to name: locating the limits of subject representation in libraries. Kluwer Academic Publishers Dordrecht, 2002.

Opengovdata.org. 8 Principles of Open Government Data. 2007. 
Ramsay, J. et al. "A psychological investigation of long retrieval times on the World Wide Web." Interacting with computers 10 (1) (1998): 77-86.

Rieusset-Lemarié, I. "P. Otlet's mundaneum and the international perspective in the history of documentation and information science." Journal of the American Society for information science 48 (4) (1997): 301-309.

Ross, S. A. "The economic theory of agency: The principal's problem." The American Economic Review 63 (2) (1973): 134-139.

Sant'ana, R. C. G. y F. Rodrigues de Assis. "Acessando dados para visualização de afinidades nas votações entre parlamentares do Senado." Informação E Sociedade: Estudos 23 (1) (2013).

Sant'ana, R. C. G. Mensuração da disponibilização de informações e do nivel de interação dos ambientes informacionais digitais da administração municipal com a sociedade. Faculdade de Filosofia e Ciências,Departamento da Ciência da Informação. Marilia: UNESP. Doutorado: 158, 2008.

—_ Tecnologia e gestão pública municipal : mensuração da interação com a sociedade. São Paulo, Cultura Acadêmica, Programa de Publicações Digitais da Pró-Reitoria de Pós-Graduação da Universidade Estadual Paulista "Júlio de Mesquita Filho" (UNESP), (2009).

Saracevic, T. "The concept of "relevance" in information science: A historical review." Introduction to information science (1970): 111-151.

"Relevance: A review of and a framework for the thinking on the notion in information science." Journal of the American Society for information science 26 (6) (1975): 321-343.

_ _Relevance: A review of the literature and a framework for thinking on the notion in information science. Part III: Behavior and effects of relevance." Journal of the American Society for information Science and Technology 58 (13) (2007): 2126-2144.

Sebastián, M. C. et al. "La necesidad de políticas de información ante la nueva sociedad globalizada." Ciência da Informação 29 (2) (2000): 22-36.

Serres, M. H. Hominescências: O começo de uma outra bumanidade. Rio de Janeiro, Bertrand Brasil, 2003.

Shneiderman, B. "Designing information-abundant web sites: issues and recommendations." International Journal of Human-Computer Studies 47 (1) (1997): 5-29.

Designing The User Interface: Strategies for Effective HumanComputer Interaction, 4/e (New Edition). Pearson Education India, 2003.

Simon, H. A. Models of bounded rationality. Cambridge, Cambridge MIT, 1997.

Spink, A. y J. Currier. "Emerging Evolutionary Approach to Human Information Behavior", en A. Spink y C. Cole, New Directions in Human Information Behavior. Dordrecht, Netherlands: Springer: 13-31, 2006 
Stephenson, W. The play theory of mass communication, Transaction Publishers, 1988.

Tamayo Gómez, C. A. et al. "Génesis del campo de Internet en Colombia: elaboración estatal de las relaciones informacionales." Signo y pensamiento 28 (54) (2009): 238-264.

Tuominen, K. y H. Savolainen. A Social Constructionist Approach to the Study of Information Use as Discursive Action. Taylor Graham, 1997.

Tuominen, K. et al. "Information Literacy as a Sociotechnical Practice." The Library Quarterly 75 (3) (July, 2005): 329-345.

Wildemuth, B. M. y D. O. Case. "Early information behavior research." Bulletin of the American Society for Information Science and Technology 36 (3) (2010): 35-38.

Williamson, O. y S. E. Masten. The economics of transaction costs. Northampton, Massachusetts: Edward Elgar, 1999.

Zipf, G. K. Human behavior and the principle of least effort: an introduction to buman ecology. Oxford, England: Addison-Wesley Pres, 1949.

Zuboff, S. "Be the friction: Our Response to the New Lords of the Rings." Frankfurter Allgemeine, Feuilleton, 2013.

Para citar este artículo:

Berrío Zapata, Cristian et al. 2016. "Comportamiento informacional y políticas públicas de información: consideraciones teóricas alrededor del caso de DATASUS en Brasil." Investigación Bibliotecológica: Archivonomía, Bibliotecología e Información 69: 19-41. http://dx. doi.org/10.1016/j.ibbai.2016.04.011 